\title{
5-ALA fluorescence-guided surgery of CNS tumors
}

\author{
Constantinos G. Hadjipanayis ${ }^{1,2}$ - Walter Stummer ${ }^{3}$ Jason P. Sheehan ${ }^{4}$
}

Published online: 22 January 2019

(c) Springer Science+Business Media, LLC, part of Springer Nature 2019

The recent Food and Drug Administration (FDA) approval of 5-ALA (5-aminovelulinic acid; Gleolan ${ }^{\circledR}$; photonamic $\mathrm{GmbH} \& \mathrm{Co}$. KG) for use as an intraoperative optical imaging agent in patients with suspected high-grade gliomas (HGGs) in 2017 catapulted fluorescence-guided surgery (FGS) as a new standard for neurosurgeons and their patients in the United States (US) [1]. This would be the first ever optical imaging agent or tool approved for use in neurosurgery in the US. Over 20 years have passed since the initial patient series by Dr. Walter Stummer describing the use of 5-ALA to help neurosurgeons identify HGGs in the surgical field [2]. A landmark multicenter, phase III randomized trial completed 13 years ago confirmed that surgeons using 5-ALA FGS as a surgical adjunct could achieve more complete resections of tumors in HGG patients and better patient outcomes than with conventional microsurgery [3].

The field of 5-ALA FGS has now been accepted globally with over 300 papers published as of 2018. With any new technology or tool used in neurosurgery, rigorous scientific analyses are required to understand the indication and limits of that technology/tool. Furthermore, combining established tools/technologies in neurosurgery with 5-ALA FGS needs to be assessed as well. In this Special Issue of the Journal of Neuro-Oncology, we have compiled the latest manuscripts

Constantinos G. Hadjipanayis

constantinos.hadjipanayis@mountsinai.org

Walter Stummer

Walter.Stummer@ukmuenster.de

Jason P. Sheehan

jsheehan@virginia.edu

1 Icahn School of Medicine at Mount Sinai, New York, NY, USA

2 Department of Neurosurgery, Mount Sinai Beth Israel, New York, NY, USA

3 Department of Neurosurgery, Universitätsklinikum Münster, Münster, Germany

4 University of Virginia Health System, Charlottesville, VA, USA by leaders in the field focusing on current applications of 5-ALA. We begin the Special Issue by focusing on the journey to FDA approval of 5-ALA for glioma surgery so that future technologies and tools can learn from the winding regulatory path that was taken for ultimate approval. An overview of established and emerging uses of 5-ALA by Diez Valle et al. is provided to set the stage for the indications and uses of 5-ALA in the CNS. Visualization technologies for 5-ALA FGS are discussed by Wei et al. to provide our readership a better understanding of how 5-ALA fluorescence is detected. Lee et al. discuss the impact of 5-ALA on extent of resection in newly diagnosed HGGs at their institution and review the literature on this systematically. Chohan et al. describe the use of 5-ALA FGS for recurrent HGGs while Michael et al. describe the effects of 5-ALA dose on the resection of glioblastoma (GBM). The combination of technologies with 5-ALA FGS and intraoperative MRI (iMRI) is discussed by Coburgher et al.

In the current Special Issue, we also discuss the use of 5-ALA FGS for other tumor types that are now being studied in neuro-oncology patients. Kamp et al. discuss the use of 5-ALA fluorescence of cerebral metastases as a prognostic factor for local recurrence and overall survival. Valdes et al. discuss the use of 5-ALA induced protoporphyrin IX fluorescence guidance in meningioma surgery. A critical review of the use of 5-ALA in the resection of pediatric brain tumors is performed by Zhang et al. The role of 5-ALA in spinal tumor surgery is reviewed by Wainwright et al. Finally, we provide a review of a new indication for 5-ALA as a therapeutic with the photodynamic therapy (PDT) of HGGs.

We would like to thank all the manuscript authors and reviewers for providing high quality manuscripts to our Special Issue on 5-ALA FGS of CNS tumors. We believe that this will be an important contribution to the literature. 


\section{References}

1. Lakomkin N, Hadjipanayis CG (2018) Fluorescence-guided surgery for high-grade gliomas. J Surg Oncol 118:356-361. https:// doi.org/10.1002/jso. 25154

2. Stummer W, Stocker S, Wagner S, Stepp H, Fritsch C, Goetz C, Goetz AE, Kiefmann R, Reulen HJ (1998) Intraoperative detection of malignant gliomas by 5 -aminolevulinic acid-induced porphyrin fluorescence. Neurosurgery 42:518-525 (discussion 525-516)

3. Stummer W, Pichlmeier U, Meinel T, Wiestler OD, Zanella F, Reulen HJ, Group AL-GS (2006) Fluorescence-guided surgery with 5-aminolevulinic acid for resection of malignant glioma: a randomised controlled multicentre phase III trial. Lancet Oncol 7:392-401. https://doi.org/10.1016/S1470-2045(06)70665-9 